\title{
Advances in Nuclear Oncology: Diagnosis and Therapy
}

E. Bombardieri, J. Buscombe, G. Lucignani, and O. Schober, eds.

London, U.K.: Informa Healthcare, 2007, 544 pages, $\$ 459.95$

This book is a review of recent advancements in the use of nuclear medicine for the diagnosis and treatment of cancer. Most of the contributing authors are established European practitioners of nuclear medicine. The audience is nuclear medicine practitioners and nonnuclear medicine specialists caring for cancer patients. The book starts with a concise and yet comprehensive chapter on cancer biology, followed by a chapter on signal targeting that is probably too short because it lists only the different targets but does not discuss molecular requirements or critical evaluations of targeting. Diagnostic applications of nuclear medicine in oncology are discussed in 15 chapters, and therapeutic applications are reviewed in 10 chapters on different types of tumors. Technical advances are then described in the last 6 chapters.

The chapters on diagnostic applications are comprehensive and up to date. Discussions of lung, colorectal, ovarian, and neuroendocrine tumors also include reviews of the sensitivities and specificities of PET reported in the literature. Other chapters only list the different methodologies, although the chapters on lung and ovarian cancer discuss applications both in initial diagnosis and in recurrence. The images are generally of good quality and reflect the different developmental stages of whole-body SPECT, PET, PET/CT, and SPECT/CT in nuclear medicine. There is some discussion of MR spectroscopy in the brain tumor chapters and of ultrasonography in other chapters.

The therapy section starts with a chapter on differentiated thyroid cancers that is cursory in scope but includes good commentaries on European guidelines. The chapter on medullary thyroid cancer therapy describes European clinical experience using radiolabeled antibodies. Although cursory in scope, the chapters on neuroendocrine cancer and lymphoma therapies provide good coverage of current strategies. Discussions of liver, colon, and brain tumors also include locoregional use of radiolabeled antibodies and of particulate radiopharmaceuticals. The discussion of ovarian cancer treatment centers on radioimmunotherapies against different mucins.

The last portion of the book consists of 6 chapters detailing technical aspects of nuclear oncology. This portion is mostly on recent advances in instrumentation, radiopharmaceuticals, and radiation dosimetry and ends with radionuclideguided therapy.

Although there is no critical evaluation of each technology or of the clinical applications, this book covers a large range of the current practice of oncologic nuclear medicine. Its references are a good resource for practitioners and for nonnuclear medicine specialists. Although not a treatise in nuclear medicine, the book has a broad scope and wealth of references that will benefit most of the intended audience.

Franklin C. Wong
University of Texas M.D. Anderson Cancer Center
Houston, Texas

COPYRIGHT ( $) 2008$ by the Society of Nuclear Medicine, Inc.

DOI: 10.2967/jnumed.108.052597 